\title{
Spontaneous rupture of lung echinococcal cyst causing anaphylactic shock and respiratory distress syndrome
}

A 21 year old woman was admitted to hospital with a persistent productive cough, dyspnoea, and fever. There was no history of sweats or haemoptysis. At admission a chest radiograph showed a $6 \mathrm{~cm}$ lesion at the base of the left lower lobe with an air fluid level (fig lA) and an additional cavitary lesion in the left apex. The blood count was within the normal range with $0.7 \%$ eosinophils.

Five days later the patient developed severe respiratory distress and her oxygen saturation on room air fell to $82 \%$. She was intubated and mechanically ventilated. At this time the eosinophil count had increased to $10 \%$. Over the following few hours the patient developed shock with a blood pressure of 85/40 and worsening hypoxaemia (saturation $75 \%$ on $100 \%$ oxygen). The mode of ventilation was changed to pressure control without significant improvement in blood oxygenation. The eosinophil count reached $16.9 \%$ (total 12800 ) and the chest radiograph showed bilateral infiltrates compatible with ARDS (fig 1B). Fibreoptic bronchoscopy with bronchoalveolar lavage (BAL) showed no evidence of bronchial obstruction. Multiple blood and BAL cultures were negative for microorganisms, but pathological examination of the BAL fluid revealed numerous echinococcal scolices (fig 2). A combined regimen of albendazole and praziquantel was started together with high dose intravenous steroids. Four weeks later she was discharged in a stable condition on albendazole for 3 months. The patient then
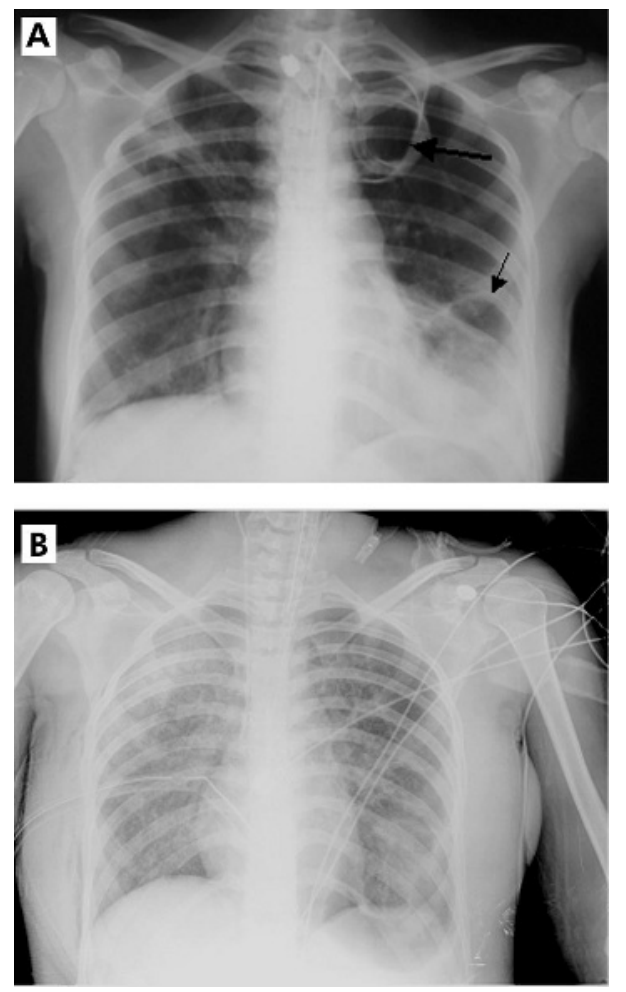

Figure 1 (A) Chest radiograph on admission. The arrows indicate the left lower and upper lobe pulmonary lesions. (B) Chest radiograph with bilateral infiltrates typical of ARDS.

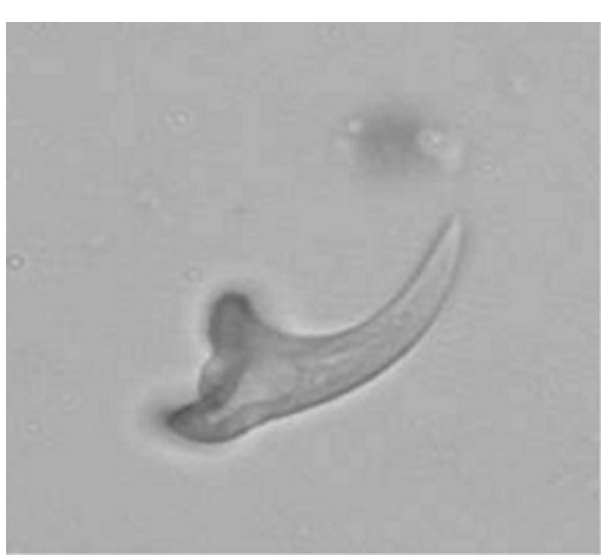

Figure 2 Echinococcal scolices in the BAL fluid.

\section{Learning point}

- Spontaneous rupture of an echinococcal cyst in the lung is a rare clinical entity and may cause anaphylactic shock and acute respiratory distress syndrome.

underwent a resection of the cyst and lobectomy of the left lower lobe with an uneventful recovery. The pathological specimen revealed a typical echinoccocal cyst.

Hydatid disease caused by Echinococcus granulosa occurs most frequently in the liver and lung. Spontaneous rupture of an echinococcal cyst is not infrequent in the liver but occurs only rarely in the lung. ${ }^{1-3}$ To our knowledge, this is the first case report of spontaneous hydatid cyst rupture into the bronchial tree with anaphylactic shock and acute respiratory distress syndrome.

R A Fanne, M Khamaisi, D Mevorach, E Leitersdorf Department of Internal Medicine B, Hadassah University Hospital and The Hebrew University - Hadassah School of Medicine, Jerusalem, Israel

M Khamaisi

Diabetes Center, Hadassah University Hospital and The Hebrew University - Hadassah School of Medicine, Jerusalem, Israel

Institute of Pulmonology, Hadassah University Hospital and The Hebrew University - Hadassah School of Medicine, Jerusalem, Israel

B Maly

Department of Pathology, Hadassah University Hospital and The Hebrew University - Hadassah School of Medicine, Jerusalem, Israel Correspondence to: Dr R A Fanne, Department of Internal Medicine B, Hadassah University Hospital and Hebrew University School of Medicine, Kiryat Hadassah, P O Box 12000, Jerusalem 911 20, Israel: arami@hadassah.org.il

\section{REFERENCES}

1 Pishori T, Azami R, Ali SM. Hydatidosis: experience with hepatic and pulmonary hydatid disease. J Pak Med Assoc 1998;48:205-7.

2 Anthi A, Katsenos C, Georgopoulou S, et al. Massive rupture of a hepatic hydatid cyst associated with mechanical ventilation. Anesth Analg 2004;98:796-7.

3 Georgiou S, Maroulis J, Monastirli A, et al. Anaphylactic shock as the only clinical manifestation of hepatic hydatid disease. Int J Dermatol 2005;44:233-5. 